\title{
LOS OBJETIVOS DEL DESARROLLO SOSTENIBLE DESDE LA PERSPECTIVA DE LA EDUCACIÓN AMBIENTAL CRÍTICA
}

\author{
OBJETIVOS DO DESENVOLMENTO SUSTENTÁVEL NA PERSPECTIVA DA EDUCAÇÃO AMBIENTAL \\ CRÍTICA
}
THE OBJECTIVES OF SUSTAINABLE DEVELOPMENT IN THE PERSPECTIVE OF CRITICAL ENVIRON- MENTAL EDUCATION

\author{
Gerson Luiz Buczenko \\ Doutor em Educação - Universidade Tuiuti do Paraná - UTP \\ Coordenador dos Cursos Superiores de Tecnologia em Segurança Pública e Gestão do Trânsito e Mobilida- \\ de Urbana, no Centro Universitário Internacional - Uninter. \\ E-mail: glbuczenko18@gmail.com
}

\begin{abstract}
RESUMEN
El propósito general del presente trabajo es analizar los objetivos del desarrollo sostenible (ODS) establecidos en septiembre de 2015, en común acuerdo suscrito por 193 países en la Asamblea General de las Naciones Unidas, desde la perspectiva de la Educación Ambiental Crítica. Como objetivos específicos se establecieron: presentar el concepto de Educación Ambiental desde la perspectiva crítica; conocer los objetivos del desarrollo sostenible; y analizar los ODS en la perspectiva de la Educación Ambiental Crítica. La cuestión planteada en la investigación fue: ¿existen aproximaciones posibles para pensar el alcance de los ODS, a través de una Educación Ambiental en su vertiente crítica? En ese sentido, este trabajo supone que la Educación Ambiental en su vertiente crítica es definida en Brasil a partir de una matriz que percibe la educación como elemento de transformación social, inspirada en el diálogo, en el ejercicio de la ciudadanía, en el fortalecimiento de los sujetos, en la superación de las formas de dominación capitalistas y en la comprensión del mundo en su complejidad. De tal manera, se evidencia que la Educación Ambiental en su vertiente crítica, emancipadora y transformadora de la realidad, se muestra como un camino viable para alcanzar los objetivos del desarrollo sostenible.
\end{abstract}

Palabras-clave: Educación. Ambiental. Crítica. ODS.

\section{RESUMO}

O propósito geral deste trabalho é analisar os objetivos do desenvolvimento sustentável (ODS) estabelecidos em setembro de 2015, em acordo assinado por 193 países, na Assembleia Geral das Nações Unidas, na perspectiva da Educação Ambiental Crítica. Como objetivos específicos estabeleceram-se: apresentar o conceito de Educação Ambiental desde a perspectiva crítica; conhecer os objetivos do desenvolvimento sustentável e analisar os ODS desde o ponto de vista da Educação Ambiental Crítica. A interrogante planteada na pesquisa foi: existem aproximações possíveis para pensar o alcance dos ODS, por meio de uma Educação Ambiental na sua vertente crítica? Nesse sentido, este trabalho supõe que a Educação Ambiental na sua vertente crítica se define no Brasil a partir de uma matriz que percebe a educação como elemento de transformação social, inspirada no diálogo, no exercício da cidadania, no fortalecimento dos sujeitos, na superação das formas de domínio capitalista e na compreensão do mundo na sua complexidade. Dessa maneira, evidencia-se que a Educação Ambiental, na sua vertente crítica, emancipadora e transformadora da realidade, se apresenta como um caminho viável para alcançar os objetivos do desenvolvimento sustentável.

Palavras-chave: Educação. Ambiental. Crítica. ODS. 


\section{ABSTRACT}

The main objective of this paper is to analyze the objectives of sustainable development (OSD) established in September 2015, in an agreement signed by 193 countries at the United Nations General Assembly, from the perspective of Critical Environmental Education. The specific objectives were: to present the concept of Environmental Education in the critical perspective; to know the objectives of sustainable development; to analyze the OSD in the perspective of Critical Environmental Education. The research question listed was: are there possible approaches to think of the achievement of OSD, through an Environmental Education in its critical aspect? In the present work we start from the assumption that Environmental Education in its critical aspect is defined in Brazil from a matrix that sees education as an element of social transformation, inspired by dialogue, in the exercise of citizenship, in the strengthening of subjects, in the overcoming capitalist forms of domination and understanding the world in its complexity. At the end, it is evident that Environmental Education, in its critical, emancipating and transforming aspect of reality, shows itself as a viable path for the pursuit of the sustainable development goals.

Keywords: Education. Environmental. Critical. OSD.

\section{INTRODUCCIÓN}

El propósito general del presente trabajo es analizar los objetivos del desarrollo sostenible establecidos en septiembre de 2015, en acuerdo suscrito por 193 países en la Asamblea General de las Naciones Unidas, desde la perspectiva de la Educación Ambiental Crítica. Como objetivos específicos se determinaron: presentar el concepto de Educación Ambiental en la perspectiva crítica; conocer los objetivos del desarrollo sostenible y analizar los ODS desde la perspectiva de la Educación Ambiental Crítica. La cuestión planteada en la investigación fue: ¿existen aproximaciones posibles para pensar el alcance de los ODS, a través de una Educación Ambiental en su vertiente crítica?

Entre los autores que sustentan este análisis están Loureiro (2002; 2004; 2012), Maia (2015), Carvalho (2004; 2012); Gadotti (2009), entre otros. La percepción inicial es que la Educación Ambiental desde su perspectiva crítica solo abarca los ODS, sin embargo, al proponerse un análisis crítico de la realidad actual, se toma en cuenta la emancipación y la transformación. La Educación Ambiental crítica está presente en todos los ODS y, más allá, defiende un nuevo panorama societario.

\section{Educación ambiental crítica}

Según Loureiro (2012, p. 28), la Educación Ambiental (EA) es definida en Brasil a 
partir de una matriz que percibe la educación como elemento de transformación social inspirada en: el diálogo, el ejercicio de la ciudadanía, el fortalecimiento de los sujetos, la superación de las formas de dominación capitalistas -a veces reproducidas en el ámbito escolar- y en la comprensión del mundo en su complejidad y de la vida en su totalidad. El diálogo es entendido en el sentido original de cambio y reciprocidad, convirtiéndose en la base de la educación.

Para Carvalho (2012), la construcción de una EA crítica impone la explicitación de algunas posiciones teórico-metodológicas; la primera de ellas con relación a la manera como se percibe la educación, que debe ser entendida como un proceso de humanización socialmente ubicado. De este modo, repensar sobre la forma como ocurre la educación es una condición prioritaria; es necesario valorar a todos los sujetos insertos en el proceso educacional, considerando que no hay más espacio para una educación bancaria, sino para la dialógica, en que el aprendizaje es mutuo e integrado al medio ambiente en que se vive. Entre los objetivos de una Educación Ambiental crítica la autora enumera:

Promover la comprensión de los problemas socioambientales en sus múltiples dimensiones: geográfica, histórica, biológica y social, considerando el medio ambiente como el conjunto de las interrelaciones entre el mundo natural y el mundo social, mediado por saberes locales y tradicionales, además de saberes científicos; contribuir para la transformación de los actuales patrones de uso y distribución de los recursos naturales, en dirección a las formas más sostenibles, justas y solidarias de relación con la naturaleza; formar una actitud ecológica dotada de sensibilidades estéticas, éticas y políticas atentas a la identificación de los problemas y conflictos que afectan el medio ambiente en el que vivimos. Involucrar a los sujetos de la educación en la solución o mejoría de estos problemas y conflictos, mediante procesos de enseñanza/aprendizaje formales y no formales que señalen la construcción significativa de conocimientos y la formación de una ciudadanía ambiental; actuar en el cotidiano escolar y no escolar, provocando nuevos cuestionamientos, situaciones de aprendizaje y desafíos para la participación en la resolución de problemas, con la finalidad de articular la escuela con los ambientes locales y regionales en donde está inserta; construir un proceso de aprendizaje significativo, conectando la experiencia y los repertorios ya existentes con cuestiones y otras experiencias que puedan generar nuevos conceptos y significados para quien se abre a la aventura de comprender el mundo que le rodea y dejarse sorprenderse por él; situar el educador, sobre todo, como mediador de relaciones socioeducativas, coordinador de acciones, investigaciones y reflexiones - escolares y/o comunitarias - que hagan posibles nuevos procesos de aprendizajes sociales, individuales e institucionales (CARVALHO, 2012, p. 160). ${ }^{1}$

1 Todas las traducciones de citas son de responsabilidad de los autores. 
Con apoyo en Carvalho (2012) y Loureiro (2002), hablar en EA crítica y transformadora es afirmar la educación como praxis social que contribuye en el proceso de construcción de una sociedad sostenible, regida por niveles de civilizaciones y sociedades distintos de los actuales, teniendo como esencia la sostenibilidad de la vida y la ética ecológica. Para Loureiro (2002), la EA transformadora es aquella que posee un contenido emancipador, en que la dialéctica entre forma y contenido se realiza de tal modo que las alteraciones de la actividad humana, vinculada al hacer educativo, implican cambios individuales y colectivos, locales y globales, estructurales y coyunturales, económicos y culturales.

Como EA emancipadora, se pueden incluir otras denominaciones como sinónimos o conceptos semejantes: “Educación Ambiental crítica; Educación Ambiental popular, Educación Ambiental transformadora" (LOUREIRO, 2012, p. 39). En esa perspectiva, el autor aun señala que es estrictamente histórico y cultural el modo como se define y se entiende la naturaleza, desde las relaciones sociales y del modo de producción y organización en determinado contexto. Si eso es ignorado, la actuación será marcadamente ingenua, sin capacidad de historiar la acción educativa, y a veces podrá hasta parecer antagónica a lo que es definido como inherente a la EA. El autor concluye que,

En una perspectiva histórica y crítica, la atribución central de la Educación Ambiental es hacer que las visiones ecológicas de mundo sean discutidas, comprendidas, problematizadas e incorporadas en todo tejido social y sus manifestaciones simbólicas y materiales, en un proceso integrador y sin imposición de una única concepción hegemónicamente vista como verdadera (LOUREIRO, 2012, p. 45).

Según Guimarães (2006), la propuesta de EA crítica se ha rotado para un proceso que revela y desconstruye los paradigmas de la sociedad moderna en sus trampas. Por otro lado, es un proceso comprometido con transformaciones de la realidad socioambiental, que pasa a construir nuevos paradigmas constituyentes de una sociedad ambientalmente sostenible y sus sujetos.

Creo que es por la praxis de una educación ambiental crítica, promotora de un movimiento colectivo conjunto que la educación y sus educadores pueden contribuir de hecho para la superación de esa grave crisis ambiental que cruzamos en nuestro pequeño planeta (GUIMARÃES, 2006, p. 27). 
Para Maia (2015), la EA crítica evidencia que la educación no puede ser instrumento ideológico a servicio de intereses mayoritarios. Todos los que trabajan en esa concepción buscan constituir sujetos históricos comprometidos con la construcción social, diferente de lo que ocurre actualmente, injusta y excluyente. El autor enfatiza en su argumento que debe haber una predisposición para el embate con el conformismo reinante en la colectividad educacional, buscando el cambio de pensamiento, el revelar verdades, así como la emancipación por el conocimiento. Es necesaria una renovación política, ética y cultural de la sociedad, interesada en el máximo desarrollo de la condición humana, rompiendo definitivamente con el actual modo de producción centrado en el neoliberalismo globalizado.

Como destaca Maia (2015), se puede entender EA, sin perder de vista la importancia del profundo rigor teórico, como proceso de recuperación de valores perdidos en la relación histórica de los seres humanos con el medio natural. Ella se desarrolla a medida que evidencia potencialidades humanas para acciones dentro de la realidad cotidiana, que favorezcan la integración del individuo corpóreo, estético, social, político, emotivo e inteligente con su alrededor, superando la dicotomía sociedad-naturaleza.

Según Tozzoni-Reis (2001), la EA es una dimensión de la educación, una actividad intencional de la práctica social, que imprime al desarrollo individual un carácter social en su relación con la naturaleza y los otros seres humanos. Así, el objetivo es potencializar esa actividad humana, convirtiéndola más plena de práctica social y de ética ambiental. Esa actividad intencional de práctica social exige una sistematización a través de una metodología que organice los procesos de transmisión y de aproximación crítica de conocimiento, actitudes y valores políticos, sociales e históricos.

[...] si la educación es mediadora en la actividad humana, articulando teoría y práctica, la Educación Ambiental es mediadora de la apropiación, por los sujetos, de las cualidades y capacidades necesarias a la acción transformadora responsable frente al ambiente en que viven. Se puede decir que la génesis del proceso educativo ambiental es el movimiento de hacerse plenamente humano por la apropiación/transmisión crítica y transformadora de la totalidad histórica y concreta de la vida de los hombres en el ambiente (TOZZONI-REIS, 2001, p. 42-43).

Según Carvalho (2004), con la perspectiva de una EA crítica, la formación incide sobre las relaciones del individuo y de la sociedad, en este sentido, individuo y colectividad 
solo tienen sentido si pensados en relaciones. Las personas se constituyen en la relación con el mundo en el que viven con los demás y por el cual son responsables por los otros. En la EA crítica, esa asunción de corresponsabilidad por el mundo supone la responsabilidad consigo mismo, con los demás y con el ambiente, es decir, con el colectivo de manera general, sin divisiones o jerarquías sobre esas dimensiones de la acción humana.

La EA crítica se propone aclarar la realidad, según Guimarães (2004) e, inscribiéndose en el proceso educativo, contribuye en la transformación de la sociedad actual; así, adquiere de forma inalienable su dimensión política. Por lo tanto, en la educación formal, ciertamente ese proceso educativo no basta dentro de los muros de una escuela. Para Loureiro (2004, p. 81), la EA

\begin{abstract}
transformadora enfatiza la educación mientras proceso permanente, cotidiano y colectivo por lo cual actuamos y reflexionamos, transformando la realidad de vida. Está enfocada en las pedagogías que problematizan el concreto vivido, en el reconocimiento de las distintas necesidades, intereses y modos de relaciones en la naturaleza que definen los grupos sociales y el 'lugar' ocupado por estos en sociedad, como medio para buscar nuevas síntesis que indiquen caminos democráticos, sostenibles y justos para todos. Se basa en el principio de que las certezas son relativas; en la crítica y autocrítica constante y en la acción política como modo de establecer movimientos emancipadores y de trasformación social que permitan el establecimiento de nuevos niveles de relaciones en la naturaleza.
\end{abstract}

La EA crítica, que transforma, libera y emancipa, se convierte en una prioridad, delante del cuadro social en que se vive y de la crisis ambiental tan pregonada en la actualidad. Ella propone el movimiento del colectivo, siendo la institución educacional uno de los grandes espacios educadores sostenibles (BORGES, 2011) para que dicho movimiento germine, en la formación de generaciones con una clara visión de que existen otras posibilidades, ante el cuadro hegemónico que se amolda y se sugiere como ideal.

La EA crítica, emancipadora y transformadora posibilita pensar en un nuevo contrato social que valore a todos los seres vivos, la identidad territorial y el sentimiento de pertenencia al medio ambiente, del cual el ser humano es parte integrante de forma armónica con los demás. 


\section{Objetivos del desarrollo sostenible}

Los ODS, conforme se ve en la figura 1, son resultado de una reunión de representantes de varios países, conjuntamente con los esfuerzos de la Organización de las Naciones Unidas (ONU). En un momento en que se adopta la Agenda 2030 para el desarrollo sostenible que incluye los ODS, con el objetivo de proponer una acción mundial, debidamente coordinada entre los gobiernos, empresas, instituciones de enseñanza -las universidadesy la sociedad civil, para alcanzar los 17 ODS y sus 169 metas. Su propósito es el de erradicar la pobreza en el mundo, promover una vida digna para todos, dentro de los límites del planeta, el cual ya emite graves señales resultantes de acción humana. La definición del desarrollo sostenible y su defensa es bastante polémica y motiva un gran debate teórico-ideológico. Para el Programa de las Naciones Unidas para el Desarrollo, el desarrollo sostenible es

definido como el desarrollo que busca satisfacer las necesidades de la generación actual, sin comprometer la capacidad de las futuras generaciones de satisfacer sus propias necesidades. Desarrollo sostenible demanda un esfuerzo conjunto para la construcción de un futuro inclusivo, resiliente y sostenible para todas las personas y todo el planeta (PNUD, 2014).

FIGURA 1 - Objetivos del Desarrollo Sostenible.

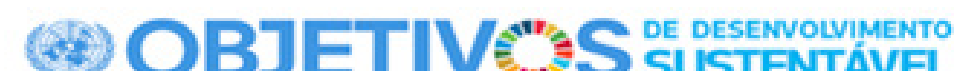
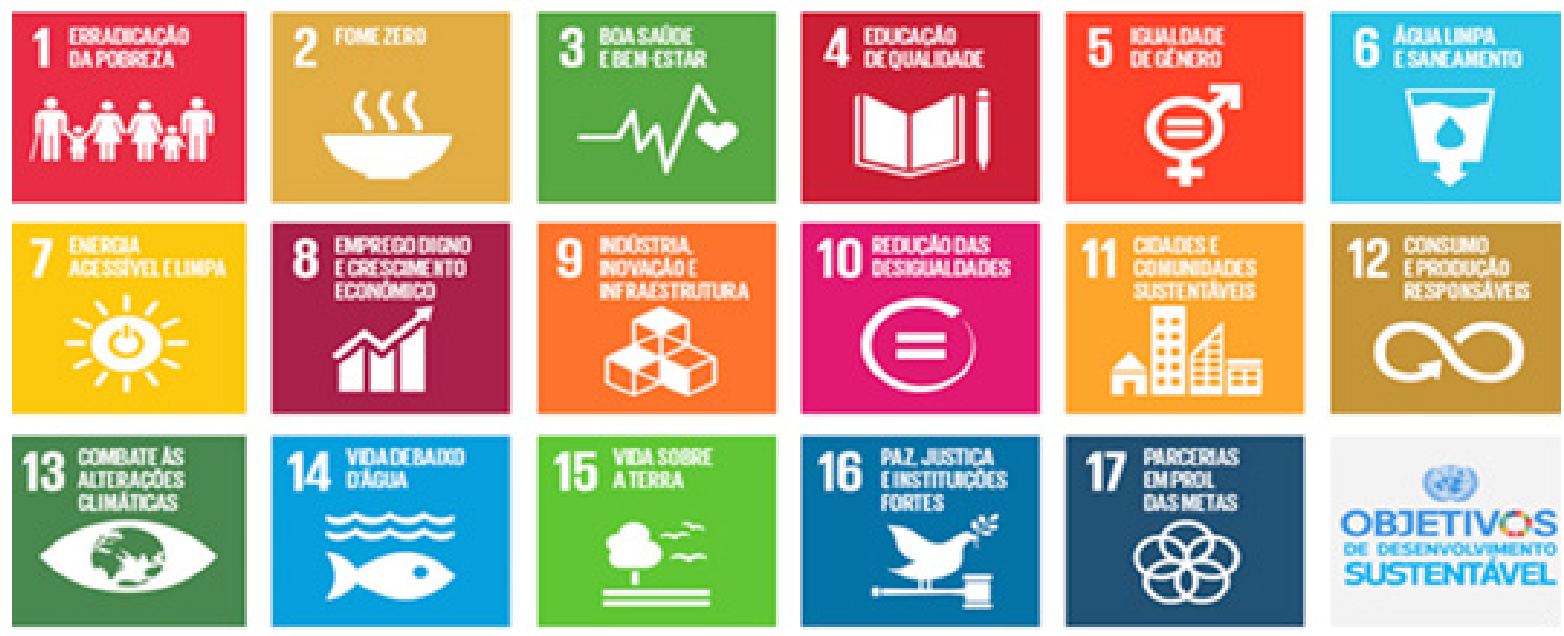

(82)

OBJETIVOS of orserwocventron
SUSTENTAVEL

Fuente: Brasil (2019).

Los ODS entraron en vigor en $1^{\circ}$ de enero de 2016 y se espera que sean cumplidos 
hasta el 31 de diciembre de 2030; existe aún la expectativa de que algunas metas, en función de ser acuerdos internacionales, sean logradas antes del plazo final. Estos objetivos no presentan un aspecto vinculante, sin embargo, se espera que las naciones se apropien de esta agenda y establezcan estrategias para lograr los 17 objetivos, que deben ser colocados como prioridades para todos los involucrados.

Trabajar para alcanzar los Objetivos Globales tornará el mundo mejor para las futuras generaciones - el mundo que ellas vivirán. Debemos disfrutar esta oportunidad para cambiar nuestro mundo para mejor. Entendemos qué podemos y debemos hacer para erradicar la extrema pobreza, el hambre y el sufrimiento innecesario, y podemos construir una comunidad mundial que provee a todos sus ciudadanos el derecho igual para que vivan sus vidas en plenitud - todo esto sin perjudicar al planeta (PNUD, 2014).

Los objetivos del desarrollo sostenible están enumerados de la siguiente forma: 1 - fin de la pobreza; 2 -hambre cero; 3 - salud y bienestar; 4 - educación de calidad; 5 - igualdad de género; 6 - agua limpia y saneamiento; 7 - energía asequible y no contaminante; 8 - trabajo decente y crecimiento económico; 9 - industria, innovación e infraestructura; 10 - reducción de las desigualdades; 11 - ciudades y comunidades sostenibles; 12 - producción y consumo responsables; 13 - acción por el clima; 14 - vida submarina; 15 - vida de ecosistemas terrestres; 16 - paz, justicia e instituciones sólidas; 17 - alianzas para lograr los objetivos.

Según Gadotti (2009, p. 49), las críticas al concepto de desarrollo sostenible y a la propia idea de sostenibilidad, vienen del hecho de que muchas veces el ambientalismo trata las cuestiones sociales aparte de las cuestiones ambientales. Por lo tanto, en virtud de ello, el autor defiende que se debe tratar de cuestiones socioambientales buscando no apartar las necesidades del planeta y las necesidades humanas. Los ecologistas,

los ambientalistas y todos nosotros necesitamos convencer a la mayoría de la población, a la población más pobre, de que no se trata solamente de limpiar los ríos, descontaminar el aire, reforestar los campos devastados para que vivamos en un planeta mejor en un futuro distante. Se trata de dar una solución, simultáneamente, a los problemas ambientales y a los problemas sociales. Los problemas tratados por la Ecología no afectan apenas el medio ambiente. Afectan al ser más complejo de la naturaleza que es el ser humano (GADOTTI, 2009, p. 50).

Por lo tanto, para Gadotti (2009, p.51), el concepto de desarrollo sostenible todavía 
es bastante polémico, además tomó y aun está tomando demasiado tiempo del debate en varios centros intelectuales del mundo, y en Brasil no es diferente. Según el mismo autor esta ambigüedad

solamente será superada en la práctica. Los debates teóricos son importantes, pero tienen un límite sin su utilización práctica. Planes teóricos proporcionarán más consistencia a la década de las Naciones Unidas para el desarrollo sostenible, superando propuestas generales. Al fin y al cabo, la sostenibilidad y el desarrollo sostenible, que proponen nuevos modos de producción y reproducción de vida nuevos modos o estilos de vida sostenibles -, dependen, en su práctica, de la correlación de fuerzas políticas existentes en la sociedad. La práctica deberá necesariamente superar la ambigüedad establecida por la "vaguedad" de los conceptos en ella presentados (GADOTTI, 2009, p.52).

\section{ODS y educación ambiental en la perspectiva crítica}

Al observar los Objetivos del Desarrollo Sostenible, a primera vista nos parece algo un tanto inalcanzable, principalmente en países como Brasil, que, además de un cuadro político bastante inestable, como una población dividida entre dos vertientes políticas, presenta atrasos en infraestructura que son de primer orden. Si pensamos en el primer objetivo, que es el fin de la pobreza, parece como mínimo imposible en los días actuales, pues la desigualdad social, en vez de retroceder, ha avanzado y tomado nuevos lares, los de las clases sociales que hasta entonces estaban fuera de la línea de pobreza, como por ejemplo, la clase media.

Otros objetivos también parecen difíciles de ser alcanzados hasta 2030, como el de la educación de calidad, ya que el país ocupa actualmente el penúltimo lugar en el ranking global de calidad de educación², superado por países como México, Tailandia y Colombia. Con relación al objetivo que trata del agua limpia y saneamiento, ocurre el mismo proceso, es decir, Brasil tiene un pasivo gigantesco, con aproximadamente 35 millones de personas que todavía no tienen acceso al agua tratada³, y nada más que la mitad de la población no

2 Ver Asociación Brasileña de Educación - "Brasil queda en penúltimo lugar en el ranking global de calidad de educación". Disponible en: http://www.abe1924.org.br/56-home/257-brasil-fica-em-penultimo-lugar-em-ranking-global-de-qualidade-de-educacao. Acceso en: 13 abr. 2019.

3 Ver Infografía: la realidad del saneamiento básico en Brasil. Disponible en: https://noticias.portaldaindustria.com.br/especiais/infografico-a-realidade-do-saneamento-basico-no-brasil/. Acceso en: 13 abr. 2019. 
tiene acceso a los servicios de drenaje y de alcantarillado.

En definitiva, son brutales los desafíos de Brasil, con relación al alcance de los objetivos del desarrollo sostenible, sin embargo, al aproximarse de la perspectiva de la EA crítica, se verifica que un camino está trazado. A la vez, dicha educación propone el trabajo constante, de forma cotidiana y colectiva, en el sentido de moverse no solamente en dirección al equilibrio en la relación ser humano y naturaleza. Implica también cuestionar la manera de ser de la sociedad que tiene como pilar consolidado el consumo desenfrenado, en la lógica de la sociedad del capital.

Así, la EA en su vertiente crítica, se coloca en la vanguardia al buscar la emancipación y la transformación de la realidad que se vive en Brasil y en otros puntos del planeta, proponiendo una profunda reflexión sobre las posibilidades de construcción de un futuro con nuevas bases, que culminen con un desarrollo sostenible para todos de forma igualitaria.

Emancipar significa "sacar las manos de"; emanciparse significa liberarse. Hay varias concepciones de educación. Ella puede ser tanto domesticadora como emancipadora. Lo que defendemos como concepción de educación es una concepción emancipadora del ser humano. Todos los seres humanos tienen derecho a desarrollar plenamente todas sus capacidades. Todos tienen derecho a una educación emancipadora (ROMÃO, 2012, p.104).

Se permite pensar que -para una sociedad debidamente educada-, manifestada la emancipación del pensamiento que busca la transformación social, el alcance de los objetivos del desarrollo sostenible sería una tarea sencilla y lógica, una vez que, además de traer beneficios a todos, ahorraría al planeta Tierra una exploración violenta, que se percibió en el pasado y que permanece en el presente.

\section{CONSIDERACIONES FINALES}

Regresando a los objetivos específicos inicialmente enumerados, los consideramos debidamente tratados en el presente trabajo, que desarrolla un abordaje profundo sobre la Educación Ambiental en su vertiente crítica. La EA crítica, de forma primordial, cuestiona el modelo hegemónico de sociedad, que presenta problemas graves, como sucede en 
Brasil, además de varios países del planeta, que enfrentan dificultades todavía más graves. Con relación a los Objetivos del Desarrollo Sostenible que fueron presentados brevemente a través de su evolución, su concepto y sus intenciones mayores por parte de las Naciones Unidas, se observa que aún se plantea un debate sobre la perspectiva del desarrollo sostenible y sus relaciones antagónicas con la lógica de la sociedad de consumo, que tiene como base el capital.

Infelizmente, parar empeorar este cuadro, muchas naciones aún presentan problemas con nacionalismos exacerbados; todavía se ven líderes mundiales que diseminan la violencia y defienden claramente la desigualdad social, además de las desgracias ya conocidas como la lucha por fuentes de energía con base en el petróleo, la exploración y la contaminación de los mares, el corte desenfrenado de las selvas entre otras tantas, que también se hacen presentes en Brasil, principalmente en el escenario político actual.

Con las dos cuestiones planteadas, se buscó una reflexión sobre las posibilidades de aproximación entre los ODS y la Educación Ambiental desde su perspectiva crítica. Se puede señalar que, a pesar de los cambios estructurales de Brasil y las vicisitudes políticas vividas en la actualidad, la Educación Ambiental desde su perspectiva crítica, emancipadora de los seres humanos y transformadora de la realidad, se presenta con un camino viable para buscar un nuevo patrón societario -que no el del capital-, haciendo posible soñar con el alcance de los objetivos del desarrollo sostenible real y para todos los pueblos del planeta.

\section{REFERÊNCIAS}

BORGES, C. O que são espaços educadores sustentáveis. In: TV ESCOLA. Espaços educadores sustentáveis. [S.I.], 2011. (Salto para o Futuro, ano XXI, 7). p. 11-16. Disponible en: http://www.nuredam.com.br/files/documentos_mec/194055espacoseducadoressustentav eis.pdf. Acceso en: 26 mar. 2019.

BRASIL. Ministério das Relações Exteriores. ODS - Objetivos do Desenvolvimento Sustentável. Disponible en: http://www.itamaraty.gov.br/pt-BR/politica-externa/desenvolvimento-sustentavel-e-meio-ambiente/134-objetivos-de-desenvolvi mento-sustentavel-ods. Acceso en: 27 mar. 2019.

CARVALHO, I. C. M. Educação ambiental crítica: nomes e endereçamentos da educação. In: LAYRARGUES, P. P. (Org.). Identidades da educação ambiental brasileira. Brasilia, DF: MMA, 2004. Disponible en: http://www.mma.gov.br/estruturas/educamb/_arquivos/livro _ ieab.pdf. Acceso en: 29 dez. 2019. 
. Educação ambiental: a formação do sujeito ecológico. 6. ed. São Paulo: Cortez,

2012.

GADOTTI, M. Educar para a sustentabilidade: uma contribuição à década da educação para o desenvolvimento sustentável. São Paulo: Editora e Livraria Instituto Paulo Freire, 2009.

GUIMARÃES, M. Armadilha paradigmática na educação ambiental. In: LOUREIRO, C. F. B.; LAYRARAGUES, P. P.; CASTRO, R. S. (Org.). Pensamento complexo, dialética e educação ambiental. São Paulo: Cortez, 2006.

LOUREIRO, C. F. B. Educação ambiental e movimentos sociais na construção da cidadania ecológica e planetária. In: LOUREIRO, C. F. B.; LAYRARGUES, P. P.; CASTRO, R. S. Educação ambiental: repensando o espaço da cidadania. São Paulo: Cortez, 2002.

. Educação ambiental transformadora. In: LAYRARGUES, P. P. (Org.). Identidades da educação ambiental brasileira. Brasília, DF: MMA, 2004. Disponible en: http://www. mma.gov.br/estruturas/educamb/_arquivos/livro_ieab.pdf. Acceso en: 29 mar. 2019.

. Trajetória e fundamentos da educação ambiental. 4. ed. São Paulo: Cortez, 2012.

MAIA, J. S. S. Educação ambiental crítica e formação de professores. Curitiba: Appris, 2015.

PROGRAMA DAS NAÇÕES UNIDAS PARA O DESENVOLVIMENTO (PNUD). Cartilha do Programa. 2014. Disponible en: https://nacoesunidas.org/pos2015/. Acceso en: 27 mar. 2019.

ROMÃO, J. E. Paulo Freire e Amílcar Cabral: a descolonização das mentes. São Paulo: Editora e Livraria Instituto Paulo Freire, 2012.

TOZZONI-REIS, M. F. C. Educação ambiental: referências teóricas no ensino superior. Interface, São Paulo, v. 5, n. 9, p. 33-50. 2001. Disponible en: http://www.scielosp.org/pdf/ icse/v5ng/03.pdf. Acceso en: 13 abr. 2019.

Recebido em: 18/09/2019

Parecer em: 06/11/2019 Aprovado em: 08/11/2019 